\title{
158. FORMACIONES DE ANTIRRHINUM MAJUS EN LOS TERRITORIOS HISPALENSES
}

\author{
Eusebio CANO, Antonio GARCÍA FUENTES, \\ Antonio CANO-ORTIZ y Ramón J. MONTILLA
}

Stands of Antirrhinum majus in the Hispalense sector

Palabras clave. Asociación, sintaxonomia, corología, sur Península Ibérica, Echio boissieriAntirrhinetum majii.

Key words. Association, syntaxonomy, chorology, south of the Iberian Peninsula, Echio boissieriAntirrhinetum majii.

Echio boissieri-Antirrhinetum majii Cano, García Fuentes, Cano Ortiz y Montilla ass nova hoc loco

[Tabla 1 sintypus inv. $\mathrm{n}^{\circ} 5$ ]

Diagnosis. Asociación caracterizada por su localización sobre margas gípsicas del termo y mesomediterráneo seco-subhúmedo del sector Hispalense, en el dominio de encinares de Paeonio coriaceae-Quercetum rotundifoliae Rivas-Martínez 1965, así como en el dominio de quejigares de Viburno tiniQuercetum fagineae Torres \& Cano in Cano, Pinto-Gómes, F. Valle, J. Torres, GarcíaFuentes, Salazar, Melendo \& Mendes 2003.

Los estudios realizados en determinados territorios de la provincia Bética, revelan que la nueva comunidad se encuentra ampliamente dominada por la especie Antirrhinum majus subsp. majus, que aparece con alta frecuencia en bordes de caminos y lugares incultos, colonizando preferentemente terraplenes a menudo con fuertes pendientes, siendo su fenología primaveral tardía; disminuyendo las características de la asociación InuloOryzopsietum, al mismo tiempo que existe un comportamiento ecológico diferente, al tratarse de margas calcáreas o bien margas gípsicas, la nueva asociación no solo presenta una ecología propia, sino también una peculiar composición florística y fisonómica, no tratándose de ecotonos, ya que los muestreos han sido realizados siguiendo criterios puramente fitosociológicos.

En la unidad biogeográfica Hispalense no aparece Lobularia maritima, pero sí las dos especies del binomen Inulo viscosaeOryzopsietum miliaceae, Inula viscosa y Piptatherum miliaceum, ya que estas últimas presentan una amplia área biogeográfica, al tratarse realmente características de la alianza Bromo-Oryzopsion miliaceae. Aunque solo hemos encontrado como diferenciales las especies Antirrhinum majus subsp. majus y Echium boissieri, esta última especie perenne presenta un gran interés al desarrollarse preferentemente sobre suelos básicos del termo y mesomediterráneo, pero que puede presentarse también sobre suelos arcillosos y presenta como área de distribución el sur de la Península Ibérica

Trabajo realizado en el marco de los proyectos «Cartografía y evaluación de los ecosistemas forestales de la provincia de Jaén. Primera Fase», y « Estudio biogeográfico de la flora de Andalucía y sus relaciones con la del norte de Marruecos». 
Tabla 1

Ass. Echio boissieri-Antirrhinetum majii Cano, García Fuentes, Cano-Ortiz y Montilla ass. nova

\begin{tabular}{|c|c|c|c|c|c|c|c|c|c|c|c|}
\hline $\mathrm{N}^{\mathrm{o}}$ de orden & 1 & 2 & 3 & 4 & 5 & 6 & 7 & 8 & 9 & 10 & 11 \\
\hline Altitud $(1=10 \mathrm{~m})$ & 63 & 74 & 79 & 52 & 60 & 49 & 52 & 70 & 64 & 59 & 65 \\
\hline Superficie $\left(\mathrm{m}^{2}\right)$ & 250 & 100 & 150 & 200 & 100 & 100 & 200 & 300 & 400 & 400 & 300 \\
\hline Cobertura \% & 90 & 80 & 70 & 70 & 90 & 70 & 90 & 100 & 60 & 100 & 100 \\
\hline Inclinación \% & 40 & 35 & 30 & 25 & 20 & 10 & 5 & 10 & - & 40 & 10 \\
\hline Orientación & $\mathrm{S}$ & $\mathrm{S}$ & SE & E & NW & $\mathrm{NE}$ & W & $\mathrm{S}$ & - & $\mathrm{N}$ & $\mathrm{S}$ \\
\hline Altura media veg. (cm.) & 90 & 80 & 70 & 80 & 80 & 90 & 110 & 90 & 50 & 140 & 150 \\
\hline Características & & & & & & & & & & & \\
\hline Antirrhinum majus & 5 & 4 & 3 & 3 & 5 & 3 & 3 & 2 & 3 & 5 & 5 \\
\hline Inula viscosa & 2 & 1 & 3 & 1 & 2 & 2 & 4 & 5 & 2 & 3 & 2 \\
\hline Piptatherum miliaceum & r. & 2 & + & 1 & 1 & + & 1 & . & + & 1 & \\
\hline Echium boissieri & + & . & 1 & + & 1 & 1 & + & + & 1 & 1 & 1 \\
\hline Moricandia moricandioides & & + & $\cdot$ & + & 1 & 1 & 1 & & + & + & 1 \\
\hline Foeniculum vulgare & + & & & 1 & 2 & + & 2 & 2 & 1 & 2 & 1 \\
\hline Daucus maximus & . & + & 1 & + & 1 & 1 & . & . & . & . & . \\
\hline Convolvulus althaeoides & . & 1 & . & + & + & + & . & & . & & . \\
\hline Dactylis hispanica & . & . & & + & . & . & . & 1 & & 1 & . \\
\hline $\begin{array}{l}\text { Ononis natrix } \\
\text { Compañeras }\end{array}$ & . & . & + & . & . & . & . & . & 2 & . & . \\
\hline Sinapis alba subsp. mairei & . & & $\cdot$ & + & + & & + & + & + & 1 & $\cdot$ \\
\hline Bromus diandrus & . & 2 & . & 1 & 2 & 2 & . & & & + & . \\
\hline Psoralea bituminosa & . & 2 & & & 1 & & . & 1 & 1 & 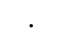 & \\
\hline Sonchus tenerrimus & . & . & + & 1 & + & 1 & . & . & . & . & . \\
\hline Medicago sativa & . & & & & + & + & . & & & . & \\
\hline Andryala integrifolia & 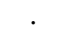 & + & + & 1 & & + & . & 1 & 1 & . & . \\
\hline Sanguisorba minor & + & + & . & 1 & 1 & + & . & . & . & . & . \\
\hline Plantago lanceolata & . & . & . & + & + & . & + & + & . & & \\
\hline Ecballium elaterium & . & . & . & + & & . & 1 & & . & 1 & 1 \\
\hline Silene vulgaris & & . & . & 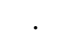 & + & . & . & + & . & + & \\
\hline Melilotus sulcata & 1 & . & . & + & 2 & . & + & . & . & 1 & + \\
\hline Eryngium campestre & . & . & . & . & + & + & . & 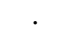 & 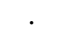 & r & . \\
\hline Centaurea pullata & . & . & & . & . & . & . & + & + & . & . \\
\hline Convolvulus arvensis & . & . & + & . & + & . & . & & . & . & . \\
\hline Allium roseum & . & . & . & & + & . & . & 1 & . & . & \\
\hline Asparagus acutifolius & 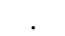 & & . & + & + & . & . & . & . & . & . \\
\hline Erodium malacoides & + & + & & + & . & . & . & . & . & . & . \\
\hline Aegilops geniculata & & & 1 & + & & 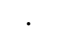 & . & . & + & 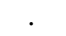 & . \\
\hline Galium parissiense & 2 & . & . & + & 2 & . & . & . & & & \\
\hline Reseda phyteuma & + & . & . & . & + & . & . & . & 1 & + & . \\
\hline Phalaris minor & r & r & & + & . & & r & . & . & + & . \\
\hline Vicia lutea subsp. vestita & 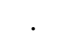 & . & 1 & . & . & + & . & 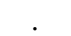 & . & . & \\
\hline Carduus tenuiflorus & + & . & . & & . & . & . & + & . & . & . \\
\hline Filago lutescens & + & 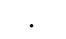 & . & 1 & . & . & . & . & . & 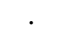 & . \\
\hline Plantago afra & . & + & . & . & . & . & . & . & . & + & \\
\hline
\end{tabular}

Además:

Lolium rigidum +, Galium aparine 2 y Valantia murale 1 en 1; Medicago polymorpha 2, Echium plantagineum +, Marrubium vulgare +, Plantago albicans + y Verbascum sinuatum + en 2; Alyssum simplex +, Avena sterilis + y Sonchus oleraceus + en 3; Geranium rotundifolium 1, Scorzonera angustifolia +, Scorpiurus muricatus +, Hypericum perforatum + y Galium verrucosum + en 4; Sisymbrium officinale +, Nigella damascena 1 y Torilis nodosa + en 5; Euphorbia helioscopia + en 6; Phagnalon saxatile 1 en 9; Papaver rhoeas + en 10 y Echinops strigosus 1 en 11.

Localidades: 1.- Carretera Jaén-Úbeda Km 45. 2.- Prox. Baeza. 3.- Prox. Torreperogil. 4.- Jaén (Polígono Llanos del Valle). 5, 7 y 8.- Carretera Jimena-Mancha Real Km 10, 12 y 16. 6.- Prox. Fuerte del Rey. 9.Prox. Jimena. 10.- Jinena-Mancha Real (cruce con Torres). 11.- Baeza-Jaén. 
Tabla 2

\begin{tabular}{|c|c|c|c|c|c|c|c|c|c|}
\hline & 1 & 2 & 3 & 4 & 5 & 6 & 7 & 8 & 9 \\
\hline Piptatherum miliaceum & $\mathrm{V}$ & IV & I & + & + & . & III & & II \\
\hline Foeniculum vulgare & IV & IV & I & . & + & . & 1 & I & I \\
\hline Psoralea bituminosa & II & II & I & ${ }^{\circ}$ & . & . & & IV & \\
\hline Convolvulus althaeoides & I & II & . & + & . & . & I & . & II \\
\hline Ditrichia viscosa & III & $\mathrm{V}$ & . & . & . & . & 1 & . & II \\
\hline Dactylis hispanica & II & I & & . & . & . & . & . & IV \\
\hline Carthamus arborescens & + & . & IV & . & . & . & . & . & \\
\hline Convolvulus arvensis & I & . & . & + & . & . & . & . & II \\
\hline Lepidium graminifolium & II & . & . & . & . & . & . & . & r. \\
\hline Cichorium intybus & I & . & . & . & . & . & . & . & + \\
\hline Centaurea aspera & $\mathrm{I}$ & . & . & . & . & . & . & . & . \\
\hline Echium asperrimum & $\mathrm{I}$ & . & . & . & . & . & . & . & . \\
\hline Aster squamatus & + & . & . & . & . & . & . & . & . \\
\hline Centaurea seridis & + & . & . & . & . & . & . & . & . \\
\hline Daucus maritimus & + & . & . & . & . & . & . & . & . \\
\hline Senecio malacitanus & + & . & . & . & . & . & . & . & . \\
\hline Beta maritima & + & . & . & . & . & . & . & . & . \\
\hline Hyparrhenia sinaica & + & & . & . & . & . & . & . & . \\
\hline Antirrhinum majus & . & $\dot{\mathrm{V}}$ & . & . & . & . & . & . & ${ }^{\circ}$ \\
\hline Echium boissieri & . & IV & . & . & . & . & . & . & $\dot{\mathrm{V}}$ \\
\hline Moricandia moricandioides & . & III & . & . & . & $i$ & & . & \\
\hline Daucus maximus & . & III & . & . & . & . & 2 & . & II \\
\hline Ononis natrix & . & I & $\dot{.}$ & $\dot{.}$ & $\dot{.}$ & . & . & . & . \\
\hline Sinapis mairei & . & II & . & . & . & . & . & . & . \\
\hline Ecballium elaterium & . & II & & . & . & . & . & . & . \\
\hline Acanthus platyphyllos & . & . & $\mathrm{V}$ & . & . & . & . & & . \\
\hline Ferula tingitana & . & $\dot{.}$ & IV & $\dot{.}$ & $\dot{.}$ & $\dot{.}$ & . & $\dot{\mathrm{V}}$ & $\dot{0}$ \\
\hline Smyrnium olusatrum & . & . & II & . & . & . & . & . & . \\
\hline Lavandula multifida & . & . & + & 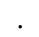 & & . & & . & . \\
\hline Hyparrhenia podotricha & . & . & I & & II & . & 2 & . & . \\
\hline Ditrichia revoluta & . & . & . & 5 & $\mathrm{~V}$ & . & . & . & . \\
\hline Oxalis pes-caprae & . & . & . & 4 & . & . & . & . & . \\
\hline Avena barbata & . & . & . & 1 & . & . & . & . & . \\
\hline Bromus matritensis & . & . & . & + & & & . & & . \\
\hline Ononis ramosissima & . & . & . & . & $\mathrm{V}$ & $\mathrm{V}$ & . & IV & . \\
\hline Verbascum litigiosum & . & . & . & . & $\mathrm{V}$ & . & . & . & . \\
\hline Seseli tortuosum & . & . & . & . & IV & & & & \\
\hline Verbascum martinezii & . & . & . & . & . & V & . & + & . \\
\hline Centaurea sonchifolia & . & . & . & . & . & + & . & . & . \\
\hline Lotus creticus & . & . & . & . & . & + & . & . & . \\
\hline Retama monosperma & . & . & . & . & . & + & & . & \\
\hline Scabiosa atropurpurea & . & . & . & . & . & I & II & . & IV \\
\hline Glaucium flavum & . & . & . & . & . & . & 1 & . & . \\
\hline Nicotiana glauca & . & . & . & . & . & . & + & . & . \\
\hline Euphorbia terracina & . & . & . & . & . & . & 1 & & . \\
\hline Carpobrotus edulis & . & . & . & . & . & . & . & $\dot{I}$ & . \\
\hline Lobularia maritima & . & . & . & . & . & . & . & I & . \\
\hline Phagnalon saxatile & . & . & . & . & . & . & . & I & ${ }^{\circ}$ \\
\hline Carlina corymbosa & . & . & . & . & 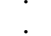 & . & . & I & $\dot{+}$ \\
\hline Chamaeleon gummifer & . & . & . & . & . & . & . & . & III \\
\hline Salvia barrelieri & . & . & . & . & . & . & . & . & I \\
\hline Scolymus maculatus & 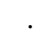 & . & . & 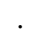 & 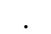 & . & . & . & III \\
\hline Notobasis syriaca & . & . & . & . & . & . & . & . & III \\
\hline Cynara humilis & . & & 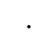 & . & . & . & . & . & IV \\
\hline Carlina racemosa & . & . & . & . & . & . & . & . & IV \\
\hline Picris echioides & & & 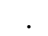 & . & . & . & 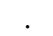 & . & II \\
\hline Scolymus hispanicus & . & . & . & . & . & . & . & . & II \\
\hline Eryngium campestre & . & . & . & 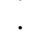 & . & . & . & . & II \\
\hline
\end{tabular}




\begin{tabular}{|c|c|c|c|c|c|c|c|c|c|}
\hline & 1 & 2 & 3 & 4 & 5 & 6 & 7 & 8 & 9 \\
\hline Anacyclus radiatus & . & . & . & . & . & . & . & . & II \\
\hline Picnomon acarna & . & . & . & . & . & . & . & . & $\mathrm{I}$ \\
\hline Carthamus lanatus & . & . & . & . & . & . & . & . & I \\
\hline Cynara baetica & . & . & . & . & . & . & . & . & + \\
\hline Ammi visnaga & . & . & . & . & . & . & . & . & + \\
\hline Phlomis herba-venti & . & . & . & . & . & . & . & . & + \\
\hline
\end{tabular}

Origen de los inventarios: 1.- Inulo viscosae-Oryzopsietum miliaceae (Bolòs, 1967; Alonso, 1996; Vicedo, 1997). 2.- Echio boissieri-Antirrhinetum majii nova (elaboración propia) 3.- Ferulo tingitanaeCarthametum arborescentes subas. carthametosum arborescentis subas. smyrnietosum olusatri (Galán et al. 2000). 4.- Inuletum revolutae (Rivas-Martínez et al.. 2002). 5.- Verbasco litigiosi-Ononidetum ramosissimae (Galán et al. 1997). 6.- Verbasco martinezii-Ononidetum ramosissimae (Galán et al.. 1997). 7.- Oryzopsio miliaceae-Daucetum maximi subas. glaucietosum flavi (Galán et al.. 2000). 8.- Verbasco martinezii-Ononidetum ramosissimae subas. feruletosum tingitanae (Galán et al. 2000). 9.- Chamaeleo gummiferi-Echietum boissieri (Müller et al. 2005).

Tabla 2 (continuación) Tabla sintética de las asociaciones pertenecientes a la alianza Bromo-Oryzopsion miliaceae y que han sido citadas en el sur de la Península Ibérica. Synthetic table of the associations included in the alliance Bromo-Oryzopsion miliaceae and cited in the south of the Iberian Peninsula.

(España y Portugal) y norte de Marruecos (Müller et al. 2005). Estos mismos autores describen para Gibraltar y norte de Marruecos la asociación Chamaeleo gummiferi-Echietum boissieri Müller, Deil, Galán de Mera \& Orellana 2005, caracterizada por la presencia de Chamaeleon gummifer, Salvia barrelieri, Echium boissieri, Delphinium pentaginum, junto a un gran grupo de especies propias de Onopordenea acanthi, como Notobasis syriaca, Cynara humilis, Carthamus lanatus, Scolymus maculatus, Scolymus hispanicus, y a otras especies meridionales como Anacyclus radiatus, no presentes en nuestros inventarios. Sin embargo la presencia de Antirrhinum majus, Ononis natrix y la alta abundancia de Piptatherum miliaceum diferencia al Echio-Antirrhinetum majii del Chamaeleo-Echietum boissieri y nos permite incluirla en la alianza Bromo-Oryzopsion miliaceae. En la Peninsula Ibérica esta alianza integra diferentes asociaciones fitosociológicas. Concretamente la asociaciacion Inulo viscosae-Oryzopsietum miliaceae O. Bolòs 1957 dominada por las especies caracteristicas Inula viscosa y Piptatherum miliaceum, fue descrita en áreas costeras de influencia termica de la subprovincia Catalana-Valenciana, provincia Catalana-Provenzal-Balear, y como su propio autor indica se introduce en los territorios mas continentalizados (Bolòs, 1967); por el contrario en el sur de la Peninsula Iberica han sido descritas diversas asociaciones, no presentándose ninguna de ellas en los territorios estudiados, así Ferulo tingitanaeCarthametum arborescentis Galán, Cortés \& I. Sánchez 2000, ha sido descrita para las zonas de Gibraltar (Galán et al. 2000), mientras que las asociaciones Verbasco litigiosi-Ononidetum ramosissimae Galán, I. Sánchez \& Vicente 1997, y Verbasco martinezii-Ononidetum ramosissimae Galán, I. Sánchez \& Vicente 1997, fueron descritas por sus autores como comunidades costeras del suroeste de la Peninsula Iberica (Galán et al. 1997), finalmente la asociación Inuletum revolutae $\mathrm{O}$. Bolòs ex Rivas-Martinez in Rivas-Martinez, E. Diaz, Fernandez-Gonzalez, Izco, Loidi, Lousa \& Penas 2002, caracterizada por el endemismo Inula viscosa subsp. revoluta ha sido descrita para el sur de Portugal (RivasMartínez et al. 2002).

La ausencia en los territorios estudiados de Ferula tingitana, Carthamus arborescens, 
Verbascum litigiosum, Verbascum martinezii, Ononis ramosissima e Inula viscosa subsp. revoluta diferencia la comunidad hispalense Echio boissieri-Antirrhinetum majii de de las asociaciones: Ferulo tingitanaeCarthametum arborescentis, Verbasco litigiosi-Ononidetum ramosissimae, Verbasco martinezi-Ononidetum ramosissimae e Inuletum revolutae. Con respecto a la asociación descrita por Bolòs y Vigo (1972) para la isla de Mallorca, y ampliada su área biogeográfica a las zonas de Gibraltar (Galán et al., 2000); (Galán et al., 2004); pensamos que no existen argumentos suficientes para traerse a los territorios gibraltareños la asociación Oryzopsio-Daucetum maximi O. Bolòs \& Vigo 1972, muy lejos del lugar donde fue descrita, ya que el taxon Daucus carota subsp. maximus, presenta un gran área de distribución al igual que las características de alianza Piptatherum miliaceum e Inula viscosa; por lo que parece más lógico interpretar esta comunidad como un resto de alguna de las asociaciones descritas en el sur peninsular (Galán et al. 1997 y 2000). La asociación Inulo viscosae-Oryzopsietum miliaceae descrita para los territorios con influencia térmica de la subprovincia Catalana-Valenciana, se encuentra dominada por las especies nitrófilas Inula viscosa, Piptatherum miliaceum y Lobularia maritima, que colonizan terrenos baldíos y bordes de caminos rurales; como su propio autor indica, se trata de comunidades bien desarrolladas en suelos más o menos profundos y arcillosos, sometidas a una fuerte insolación y sequedad, que se localizan en los dominios de Viburno tini-Quercetum ilicis (Br.-Bl ex Moliner 1934) Rivas-Martínez 1975, Quercetum rotundifoliae Br.-Bl. \& O. Bolòs in Vives 1956, Querco cocciferae-Lentiscetum Br.-B1., Font Quer, G. Br.-B1., Frey, Jansen \& Moor 1936 y en Myrto communis-Pistacietum lentisco (Moliner 1954) Rivas-Martínez 1975 (tab. 2).

\section{ESQUEMA SINTAXONÓMICO}

ARTEMISIETEA VULGARIS Lohmeyer,

Preising \& Tüxen ex von Rochow 1951

ARTEMISIENEA VULGARIS

+ Agropyretalia repentis Oberdorfer, Müller \& Görs in Oberdorfer, Görs, Komeck, Lohmeyer, Müller, Philippi \& Seibert 1967

* Bromo-Oryzopsion miliaceae O. Bolòs 1957

Echio boissieri-Antirrhinetum majii ass. nova

\section{BIBLIOGRAFÍA}

BOLÒS, O. -1967-. Comunidades vegetales de las comarcas próximas al litoral situadas entre los ríos Llobregat y Segura. Mem. Real Acad. Ci. Barcelona 38(1):3-281

BOLÒS , O. \& J. VIGO -1972-. Contribution à la géobotanique de l'ile de Majorque. Rapp. Com. Int. Mer. Médit. 21(3):81-82.

GALÁN DE MERA, A., I. SÁNCHEZ GARCÍA \& J. A. VICENTE ORELLANA -1997-. Coastal plant communities of the sothwestern Iberian Peninsula, Spain and Portugal. Phytocoenologia 27(3): 313-352

GALÁN DE MERA, A., J.E. CORTÉS e I. SÁNCHEZ GARCÍA -2000-. La vegetación del Peñon de Gibraltar. Acta Bot. Malacitana 25:107-130

GALÁN DE MERA, A., U. DEIL, A. VICENTE ORELLANA \& J.V. MÜLLER -2004Roadside vegetation in the campo the Gibraltar (SW Spain) and on the Tangier Peninsula (NW Marocco). Stud. Bot. 23:63-93

MÜLLER, J.V., U. DEIL, A. GALÁN DE MERA y A. VICENTE ORELLANA. -2005-Echium boissieri Steud. (Boraginaceae), and IberoMauretanian roadside plant. Feddes Repertorium 116 (3-4): 226-242.

Aceptado para su publicación en julio de 2006

Dirección de los autores. Dpto. Biología Animal, Vegetal y Ecología. Botánica. Universidad de Jaén. Campus Universitario. Las Lagunillas s/n. 23071 Jaén.ecano@ujaen.es 